\title{
Characterization and Evaluation of $\alpha$-Mangostin-loaded Film-forming Gels for Acne Treatment
}

\begin{abstract}
P. TANNGOEN, S. LAMLERTTHON ${ }^{1,2}$ AND W. TIYABOONCHAI ${ }^{2,3^{*}}$
Department of Pharmaceutical Technology, Faculty of Pharmaceutical Sciences, ${ }^{1}$ Department of Microbiology and Parasitology, Faculty of Medical Sciences, ${ }^{2}$ The Center of Excellence in Medical Biotechnology, Naresuan University, Phitsanulok, ${ }^{3}$ The Center of Excellence for Innovation in Chemistry (PERCH-CIC), Commission on Higher Education, Ministry of Education, Bangkok, Thailand
\end{abstract}

Tanngoen et al.: Evaluation of AM-FFGs Against Acne-causing bacteria

In this study, $\alpha$-mangostin, extracted from mangosteen peel, was developed in to a topical film-forming gel antiacne preparation using different film-forming polymers as carriers. The physicochemical properties and $\alpha$-mangostin incorporation efficiency were evaluated. In vitro permeation of $\alpha$-mangostin-loaded film-forming gels, antibacterial activity against Propionibacterium acnes and Staphylococcus aureus were examined. The optimal formulations were viscous gels, which could convert into a dry film within 30 min after application. The films were transparent, flexible and easy to peel off. Scanning electron micrographs of the $\alpha$-mangostin-loaded film-forming gels revealed a rough surface with an interior porous structure while blank film-forming gels showed smooth and compact morphology. The high $\alpha$-mangostin incorporation efficiency up to $96 \%$ was achieved. In vitro permeation studies showed a biphasic permeation profile with a fast permeation characteristic within 30 min followed by a slow permeation up to $8 \mathrm{~h}$. $\alpha$-Mangostinloaded film-forming gels demonstrated efficient antibacterial activities against $P$. acnes and $S$. aureus. Moreover, $\alpha$-mangostin-loaded film-forming gels showed good physicochemical stability during storage at $4^{\circ}$ for 6 mo. In summary, the developed $\alpha$-mangostin-loaded film-forming gels are good candidates for topical antiacne treatments.

Key words: Acne vulgaris, Propionibacterium acnes, Staphylococcus aureus, acne treatment, alphamangostin, film-forming gels

Acne vulgaris is a common problem throughout the world. The four factors involved in acne formation are the excess sebum production of sebaceous glands, follicular hyperkeratinization, follicular colonization by Propionibacterium acnes and the inflammation caused by normal skin flora such as Staphylococcus aureus $^{[1]}$. Typically initial treatment is topical with azelaic acid, salicylic acid, benzoyl peroxide, retinoids and antibiotics that can lead to undesirable side effects $^{[2]}$. Thus, there is much interest in the use of natural products with fewer side effects. $\alpha$-Mangostin (AM), extracted from mangosteen peel, is a natural product of particular interest that could benefit acne treatment. Several studies reported that AM is effective against $P$. acnes and $S$. aureus. In addition, it possesses antiinflammatory and antioxidant properties ${ }^{[3-6]}$.

Current topical formulations used to treat acne vulgaris include creams, gels, solutions, ointments or

*Address for correspondence

E-mail: wareet@nu.ac.th

January-February 2020 patches $^{[2]}$. However, their effects are often limited ${ }^{[7]}$. To overcome these limitations, one interesting delivery system is film-forming gel (FFG). FFG is a mixture of polymers dispersed homogenously in volatile solvents. It is applied to the skin as a gel, but then can form a transparent, barely noticeable film in situ as the solvent evaporate and can be easily peeled off when desired. The advantages of FFG are ease of application, minimal skin irritation, prevention or treatment of secondary bacterial infections, enhanced drug release and reduced application frequency due to a longer duration of delivery ${ }^{[8,9]}$. However, most of FFGs contain high amount of ethanol that could lead to skin irritation ${ }^{[7]}$.

This is an open access article distributed under the terms of the Creative Commons Attribution-NonCommercial-ShareAlike 3.0 License, which allows others to remix, tweak, and build upon the work non-commercially, as long as the author is credited and the new creations are licensed under the identical terms

Accepted 20 December 2019

Revised 02 October 2019

Received 24 Juy 2019

Indian J Pharm Sci 2020;82(1):157-165 
Thus, this study aimed to develop an AM-loaded FFGs (AM-FFGs) with low ethanol content. The physicochemical properties and AM incorporation efficiency of the FFGs were characterized. In vitro permeation study was performed with a Franz diffusion cell. The antibacterial activities against $P$. acnes and $S$. aureus were evaluated. Also, the physicochemical stability of prepared AM-FFGs was evaluated.

\section{MATERIALS AND METHODS}

AM was purchased from Chromadex, Inc. (Irvine, CA, USA). Polyvinyl alcohol (PVA) was purchased from Bang Trading 1992 Co. (Bangkok, Thailand). Eudragit ${ }^{\circledR}$ NE 30D was received as a free sample from Evonik Degussa Pvt. Ltd. (Mumbai, India). Kollidon ${ }^{\circledR}$ 90F (polyvinyl pyrrolidone K90) was received as a gift from BASF Ltd. (Ludwigshafen, Germany). Carbopol 934 and sorbitol were purchased from Namsiang Co., Ltd. (Bangkok, Thailand). Methanol, acetonitrile and $85 \%$ O-phosphoric acid were all HPLC grade. Other chemicals and solvents were of analytical grade. P. acnes DMST 14916 and S. aureus ATCC 25923 were obtained from the Department of Medical Sciences, Thailand.

\section{Preparation of AM-FFGs:}

In a preliminary study, nine blank FFGs were prepared by mixing $1 \%(\mathrm{w} / \mathrm{w})$ carbopol with $3.3 \%(\mathrm{w} / \mathrm{w})$ film-forming polymers, in different combinations and ratios and $2 \%(\mathrm{w} / \mathrm{w})$ plasticizer, with a total amount of ethanol $10 \%(\mathrm{w} / \mathrm{w})$. The combinations of film formers were as followed; PVA and Eudragit ${ }^{\mathbb{B}}$ NE 30D, PVA and Kollidon ${ }^{\circledR}$ 90F and Eudragit ${ }^{\circledR}$ NE 30D and Kollidon $^{\circledR} 90 \mathrm{~F}$ at the weight ratio of $1: 1,1: 2$ and $2: 1$, respectively. Before mixing, PVA and Kollidon ${ }^{\circledR}$ 90F were dissolved in water, while Eudragit ${ }^{\circledR}$ NE 30D was dissolved in ethanol. After homogeneously mixed, the $\mathrm{pH}$ of gels was adjusted to $6.0 \pm 0.5$ with $0.1 \mathrm{~N} \mathrm{NaOH}$ before further physical characterization.

Based on the physical characteristics of blank FFGs, only two optimal FFG formulations were selected for loading AM (0.125\% w/w); AM-FFG1 and AM-FFG2. AM-FFG1 was prepared with PVA and Eudragit ${ }^{\circledR}$ NE $30 \mathrm{D}$ at a weight ratio of $2: 1$, while AM-FFG2 was prepared with PVA and Kollidon ${ }^{\circledR} 90 \mathrm{~F}$ at a weight ratio of 2:1. AM was dissolved in ethanol before adding to the mixed solution as described before. These two formulations were used throughout the rest of the study, along with their two unloaded equivalents.

\section{Physicochemical characterization:}

The appearance of each sample was visually evaluated for homogeneity. The $\mathrm{pH}$ was measured using a $\mathrm{pH}$ meter (Delta 320, Mettler-Toledo, Shanghai, China). The sample viscosity was measured using a cone/plate viscometer (Model DV-II, Brookfield, Middleboro, MA, USA) with a CP-40 spindle, at $50 \mathrm{rpm}$ for $1 \mathrm{~min}$.

A drying time test was carried out to determine the time required by each gel to convert into film. About $200 \mathrm{mg}$ of each gel was spread onto a Teflon sheet mold $(2 \times 5 \mathrm{~cm} \times 0.1 \mathrm{~mm})$ and dried at $32 \pm 1^{\circ}$ in a hot air oven (Model 500, Memmert, Schwabach, Germany). Samples were checked at $5 \mathrm{~min}$ intervals during the drying process and the elapsed time at which each sample turned to film was recorded.

The light transparency of the films was measured as percent transmittance using conditions similar to those of Zhang et al. ${ }^{[10]}$. Each film was cut and stuck to the outside of a cuvette. Then its transmittance was determined using an UV/Vis spectrophotometer (Genesys 10 Series, Thermo Fisher Scientific, Inc, USA; UV/Vis) with transmission mode at a wavelength of $600 \mathrm{~nm}$.

The thickness of the films was determined using thickness gauge. Dried film sample was cut and peeled off from the Teflon sheet mold. The thickness was determined from three different points on the film. Ultimate tensile strength $\left(\mathrm{N} / \mathrm{mm}^{2}\right)$ and percentage of elongation at break were determined using a TA.XT plus C Texture Analyser (Stable Micro Systems Ltd, Surrey, UK). Dried film samples were cut $(2 \times 5 \mathrm{~cm}$ $\times 0.1 \mathrm{~mm})$ and attached to the machine jaw. The mechanical test was performed using a $5 \mathrm{~kg}$ load cell at a speed of $20 \mathrm{~mm} / \mathrm{s}$.

For the determination of AM incorporation efficiency, $200 \mathrm{mg}$ of AM-FFG films were cut into small pieces, soaked in $10 \mathrm{ml}$ of methanol and centrifuged at $18000 \mathrm{rpm}$ for $30 \mathrm{~min}$. The amount of AM in the supernatant was determined using a UV/Vis spectrophotometer at a wavelength of $320 \mathrm{~nm}$. Percent AM loading was calculated following Eqn. $1, \mathrm{AM}$ incorporation efficiency $(\%)=\mathrm{AM}$ tested $/ \mathrm{AM}$ initial $\times 100$.

The morphology of tested films was characterized using a field emission scanning electron microscope (FE-SEM: Carl Zeiss Microscopy, Jena, Germany). All films were cut into small pieces with a platinum razor blade and fixed onto a stub. Films were coated with 
gold using a sputter coater before subjected to FE-SEM for examining the film surface and internal structure. The crystallinity of blank FFG films and AM-FFG films were characterized using an X-ray diffractometer (D8 Advance, Bruker AXS Inc., Fitchburg, WI; XRD). Multiple 2-theta scans were performed within the range of 10 to $60^{\circ}$ at a speed of $0.2^{\circ} / \mathrm{min}$.

\section{In vitro permeation:}

The AM permeation profile was examined using porcine ear epidermis in a vertical Franz diffusion cell (PermaGear, Hellertown, USA) as per Pan-on et al. method with some modification ${ }^{[1]}$. Porcine ear skin was obtained from the local slaughter house and the subcutaneous layer was removed. Then, the porcine ear epidermis was separated from the dermis by heat separation technique. The separated epidermis $(25 \times 25 \mathrm{~mm})$ was placed directly between a donor and receptor compartment. The receiver compartment was then filled with $12 \mathrm{ml}$ of deionized water containing $2 \%$ Tween 80 , at a system temperature of $32 \pm 1^{\circ}$ with constant stirring. After waiting $30 \mathrm{~min}$ for the skin temperature to equilibrium, AM-FFGs containing AM $250 \mu \mathrm{g}$ was spread on the porcine epidermis. $500 \mu \mathrm{l}$ of receiver medium was collected at a set time intervals $(30,60,120,240$ and $480 \mathrm{~min})$ and immediately replaced with fresh medium. The AM content was analyzed using high performance liquid chromatography (HPLC). The accumulated AM in the porcine epidermis was also determined. Briefly, the epidermis was cut into smaller pieces, soaked in $1 \mathrm{ml}$ of methanol, and centrifuged at $18000 \mathrm{rpm}$ for $30 \mathrm{~min}$. The supernatant was then analyzed with HPLC to determine the amount of AM. Porcine ear epidermis was used in this research and it has been approved by animal ethics committee of Naresuan University, Phitsanulok, Thailand (No.NU-AEE610301).Nohumans/animalswereused for studiesin thisresearch. TheHPLC conditionsweresimilar to those of Pothitirat et al. with some modification ${ }^{[12]}$. The HPLC was equipped with a Vertisep C18 column $(250 \times 4.6 \mathrm{~mm}, 5 \mu \mathrm{m})$. The mobile phase consisted of a mixture of $0.2 \%$ aqueous o-phosphoric acid solution and acetonitrile at a ratio of $1: 9(\mathrm{v} / \mathrm{v})$. The flow rate was set at $1 \mathrm{ml} / \mathrm{min}$ with a detection wavelength of $320 \mathrm{~nm}$. The AM content was quantified using a calibration curve with a concentration ranging from 0.25 to $50 \mu \mathrm{g} / \mathrm{ml}$.

\section{Antibacterial activity:}

The antibacterial activity of AM-FFGs against P. acnes DMST 14916 and S. aureus ATCC 25923 were determined using the agar-well diffusion method as described by Balouiri et al. with some modifications ${ }^{[13]}$. An inoculum of each bacterium $\left(10^{8} \mathrm{CFU} / \mathrm{ml}\right)$ was prepared and swabbed over the entire surface of brain heart infusion agar for P. acnes and Muller-Hinton agar for $S$. aureus. Then, 4 holes were made aseptically using a sterile cork borer $(\varnothing=7 \mathrm{~mm})$. After that, $50 \mu \mathrm{l}$ of blank FFGs and AM-FFGs were added into the resulting wells. The plates containing $P$. acnes were incubated for $72 \mathrm{~h}$ at $37 \pm 2^{\circ}$ under anaerobic conditions, while the plates with $S$. aureus were incubated for $24 \mathrm{~h}$ also at $37 \pm 2^{\circ}$. The zones of inhibition of all the plates were measured in $\mathrm{mm}$.

\section{Stability study:}

The samples were stored in well-closed containers and kept in the darkness at room temperature and $4^{\circ}$ for 6 mo. Samples were withdrawn at 3 and 6 mo to examine the viscosity and AM content.

\section{Statistical analysis:}

All tests were performed in triplicate and the resulting data is expressed as the mean \pm standard deviation (SD).

\section{RESULTS AND DISCUSSION}

Nine blank FFGs were prepared with different combinations and ratios of film-forming polymers. Their physicochemical properties are shown in Table 1. F2 and F9 demonstrated heterogeneous system, so they were removed from further testing. Apart from F2 and F9, all formulations showed translucent homogeneous gels with viscosity ranging from 900-1800 cP. In addition, they manifested a $\mathrm{pH}$ value of $\sim 5.7$, which similar to the normal skin's $\mathrm{pH}$ of 5.4-5.9 $9^{[14]}$. For film characterizations, gels all converted into film within 30-40 min. All the films were high transparency, with transmittance value in the range of 70-95\%. Despite all the gels' general similarities, only F3 and F6 were selected for loading with AM. They were chosen because of their high transmission value of $>90 \%$, and drying time of $30 \mathrm{~min}$. Moreover these two FFG formulations contain a lower amount of ethanol compared to other topical products currently available, which could help reduce skin irritation while still keeping drying time reasonably short.

The physicochemical properties of blank FFGs and AMFFGs are shown in Table 2. In gel characterizations, the blank-FFG1 demonstrated translucent homogeneous gel while blank-FFG2 showed transparent homogeneous gel. The different polymer used has a direct impact on the gel clarity depending on its solubility. FFG2 contained a water soluble Kollidon ${ }^{\circledR} 90 \mathrm{~F}$, and thus, 


\begin{tabular}{|c|c|c|c|c|c|}
\hline \multirow{2}{*}{ Formulation } & \multicolumn{3}{|c|}{ Gel form } & \multicolumn{2}{|c|}{ Film form } \\
\hline & Visual appearance & $\mathrm{pH}$ & Viscosity (cP) & Drying time (min) & Transmission (\%) \\
\hline \multicolumn{6}{|c|}{ PVA: Eudragit ${ }^{\circledR}$ NE 30D } \\
\hline F1 (1:1) & homogeneous & $5.73 \pm 0.05$ & $1190 \pm 16$ & 30 & $87 \pm 2$ \\
\hline $\mathrm{F} 2(1: 2)$ & heterogeneous & N/A & N/A & N/A & $\mathrm{N} / \mathrm{A}$ \\
\hline F3 $(2: 1)$ & homogeneous & $5.55 \pm 0.07$ & $1756 \pm 7$ & 30 & $92 \pm 1$ \\
\hline \multicolumn{6}{|c|}{ PVA: Kollidon ${ }^{\circledR} 90 \mathrm{~F}$} \\
\hline F4 (1:1) & homogeneous & $5.73 \pm 0.13$ & $1565 \pm 21$ & 40 & $84 \pm 2$ \\
\hline F5 (1:2) & homogeneous & $5.60 \pm 0.11$ & $1001 \pm 19$ & 40 & $80 \pm 2$ \\
\hline F6 (2:1) & homogeneous & $5.83 \pm 0.07$ & $1270 \pm 19$ & 30 & $95 \pm 1$ \\
\hline \multicolumn{6}{|c|}{ Eudragit ${ }^{\circledR}$ NE 30D: Kollidon ${ }^{\circledR} 90 \mathrm{~F}$} \\
\hline F7 (1:1) & homogeneous & $5.64 \pm 0.07$ & $940 \pm 6$ & 30 & $83 \pm 1$ \\
\hline F8 (1:2) & homogeneous & $5.66 \pm 0.4$ & $958 \pm 13$ & 30 & $76 \pm 2$ \\
\hline F9 $(2: 1)$ & heterogeneous & $\mathrm{N} / \mathrm{A}$ & $\mathrm{N} / \mathrm{A}$ & $\mathrm{N} / \mathrm{A}$ & $\mathrm{N} / \mathrm{A}$ \\
\hline
\end{tabular}

${ }^{*} \mathrm{~N} / \mathrm{A}$ is not available. The results are presented as mean $\pm S D ; n=3$

TABLE 2: THE EFFECT OF AM INCORPORATION ON THE PHYSICOCHEMICAL PROPERTIES OF BLANK FFGS AND AM-FFGS

\begin{tabular}{|c|c|c|c|c|}
\hline \multirow{2}{*}{ Characterization } & \multicolumn{4}{|c|}{ Formulation } \\
\hline & Blank FFG 1 & AM-FFG 1 & Blank FFG 2 & AM-FFG 2 \\
\hline \multicolumn{5}{|c|}{ Gel form } \\
\hline Visual appearance & $\begin{array}{l}\text { Translucent and } \\
\text { homogenous }\end{array}$ & $\begin{array}{l}\text { Yellow, translucent } \\
\text { and homogenous }\end{array}$ & $\begin{array}{l}\text { Transparent and } \\
\text { homogenous }\end{array}$ & $\begin{array}{l}\text { Yellow, transparent } \\
\text { and homogenous }\end{array}$ \\
\hline $\mathrm{pH}$ & $5.55 \pm 0.07$ & $5.63 \pm 0.13$ & $5.83 \pm 0.07$ & $5.82 \pm 0.04$ \\
\hline Viscosity (cP) & $1756 \pm 7$ & $1738 \pm 34$ & $1270 \pm 19$ & $1201 \pm 27$ \\
\hline \multicolumn{5}{|c|}{ Film form } \\
\hline Drying time (min) & 30 & 30 & 30 & 30 \\
\hline Thickness (mm) & $0.01 \pm 0.002$ & $0.01 \pm 0.002$ & $0.01 \pm 0.003$ & $0.01 \pm 0.002$ \\
\hline Transmission (\%) & $92 \pm 1$ & $90 \pm 2$ & $95.00 \pm 1$ & $95 \pm 2$ \\
\hline $\begin{array}{l}\text { Ultimate tensile strength }(\mathrm{N} / \\
\left.\qquad \mathrm{mm}^{2}\right)\end{array}$ & $1.32 \pm 0.04$ & $1.33 \pm 0.16$ & $2.11 \pm 0.05$ & $2.15 \pm 0.11$ \\
\hline Elongation at break (\%) & $204 \pm 9$ & $211 \pm 18$ & $178 \pm 5$ & $190 \pm 28$ \\
\hline AM incorporation efficiency (\%) & $\mathrm{N} / \mathrm{A}$ & $97 \pm 1$ & $\mathrm{~N} / \mathrm{A}$ & $96 \pm 1$ \\
\hline
\end{tabular}

${ }^{*} \mathrm{~N} / \mathrm{A}$ is not available. The results are presented as mean $\pm S D ; n=3$

resulting in a greater gel clarity than FFG1 containing Eudragit $^{\circledR}$ NE 30D, which is an aqueous dispersion of a neutral copolymer and insoluble in water. For film characterization, both FFGs converted into film within $30 \mathrm{~min}$. These possessed a film thickness of $0.01 \mathrm{~mm}$ and high transparency with transmittance value of $92-95 \%$. Accordingly, as shown in figs. 1A and $\mathrm{B}$, the appearance of film was very thin, clear and almost unnoticeable, which would please users after application. In addition, there was no difference in mechanical properties between FFG1 and FFG2. These 2 formulations possessed ultimate tensile strength and $\%$ elongation at break in the range of 1.32-2.11 $\mathrm{N} / \mathrm{mm}^{2}$ and $178-204 \%$, respectively. This revealed that the prepared films were flexible and easy to peel off. The AM-FFGs showed similar physicochemical characteristics compared to their blank counterpart indicating that loading of AM in fact does not alter the FFG properties. In addition, the high AM incorporation efficiency, 96-97\%, was observed.
The morphology of blank FFG and AM-FFG were characterized using FE-SEM. The blank FFG1 and FFG2 illustrated similar morphology both of top-view and cross-sectional view, figs. $2 \mathrm{~A}$ to $\mathrm{D}$, suggesting that the different film former used in these formulation has no effect on their morphology. On the contrary, addition of AM considerably affects the film morphology. The images clearly showed that AM-loaded films, figs. 2E and $\mathrm{G}$, have a rougher texture than its blank FFG films, figs. $2 \mathrm{~A}$ and $\mathrm{C}$. These results correlate with the crosssectional images, figs. $2 \mathrm{~F}$ and $\mathrm{H}$, demonstrating the $\mathrm{AM}$ was distributed homogeneously over the surface and also entrapped in the porous structure of both films.

The XRD patterns of AM powder, blank FFG films and AM-FFG films are shown in figs. 3A and B. Generally, the high intensity and sharpness of the peak indicate a crystalline nature ${ }^{[15,16]}$. The AM showed intense peak at diffraction angle of 2-theta at 12.17, 13.51, 16.67, 19.36, 23.77, 26.49 and $30.96^{\circ}$, indicating crystalline form. 
The XRD pattern of PVA and Kollidon ${ }^{\circledR}$ 90F displayed a broad peak, while Eudragit ${ }^{\mathbb{B}}$ NE 30D showed no peak. This implied that they all are amorphous in nature. Both of blank FFGs were an amorphous nature as evidenced

(A)

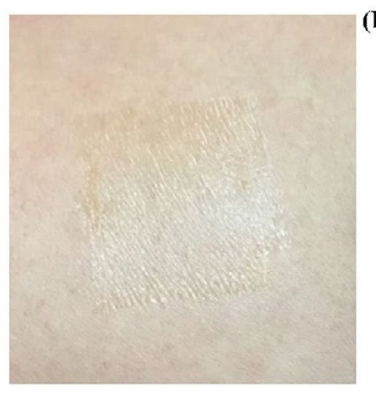

Fig. 1: The appearance of AM-FFG film

The appearance of AM-FFG film (A) after 30 min applied on the skin and (B) peeling off from the skin from the broad peak with low intensity at 16.81 and $29.68^{\circ}$ (2-theta). Interestingly, the characteristic peak of AM was only absent in the AM FFG-1 film suggesting that AM incorporated in the film as an amorphous molecular dispersion, fig. 3A. On the other hand, AMFFG2 showed low intensity AM peaks at 13.51 and $16.67^{\circ}$ (2-theta), indicating a partially crystalline nature of incorporated AM, fig. 3B.

The difference in AM polymorph found in both films could be explained by the solubility properties of the $\mathrm{AM}$ and film formers. AM and Eudragit ${ }^{\circledR}$ NE 30D are soluble in ethanol, while Kollidon ${ }^{\circledR} 90 \mathrm{~F}$ is soluble in water. Therefore, during the solvent evaporation process, AM-FFG1, composed of Eudragit ${ }^{\mathbb{B}} \mathrm{NE} 30 \mathrm{D}$, was solidified in a short time period than AM-FFG2,
Top-view
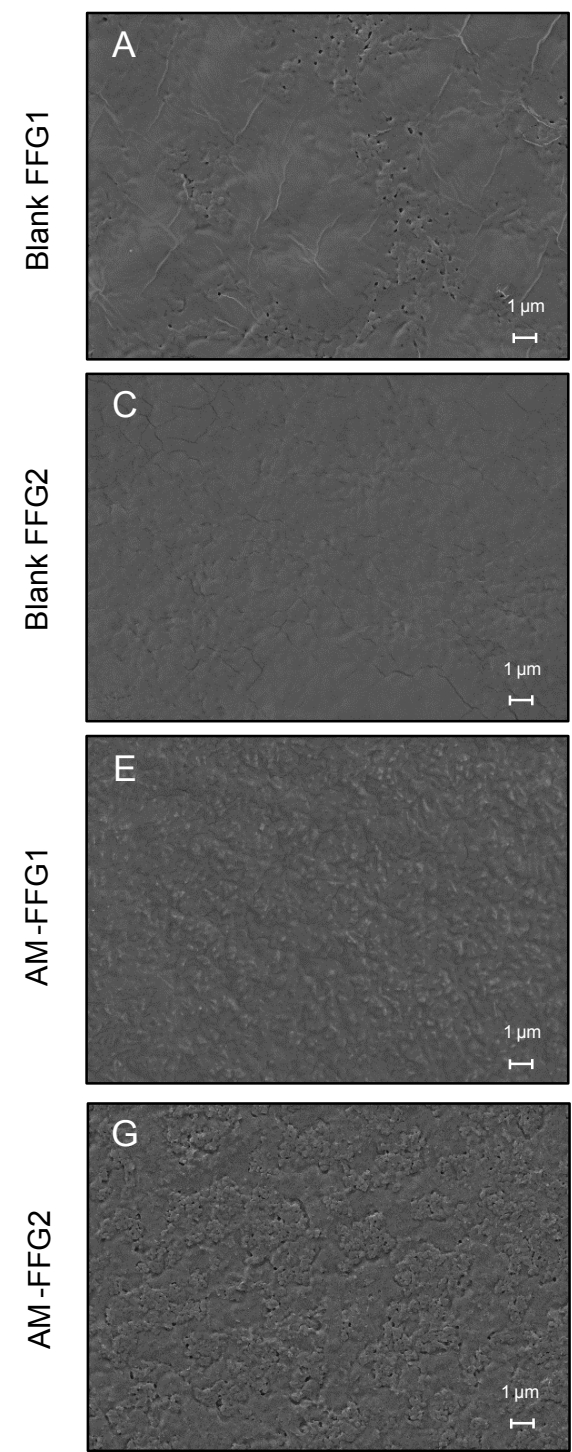

Crosssectional
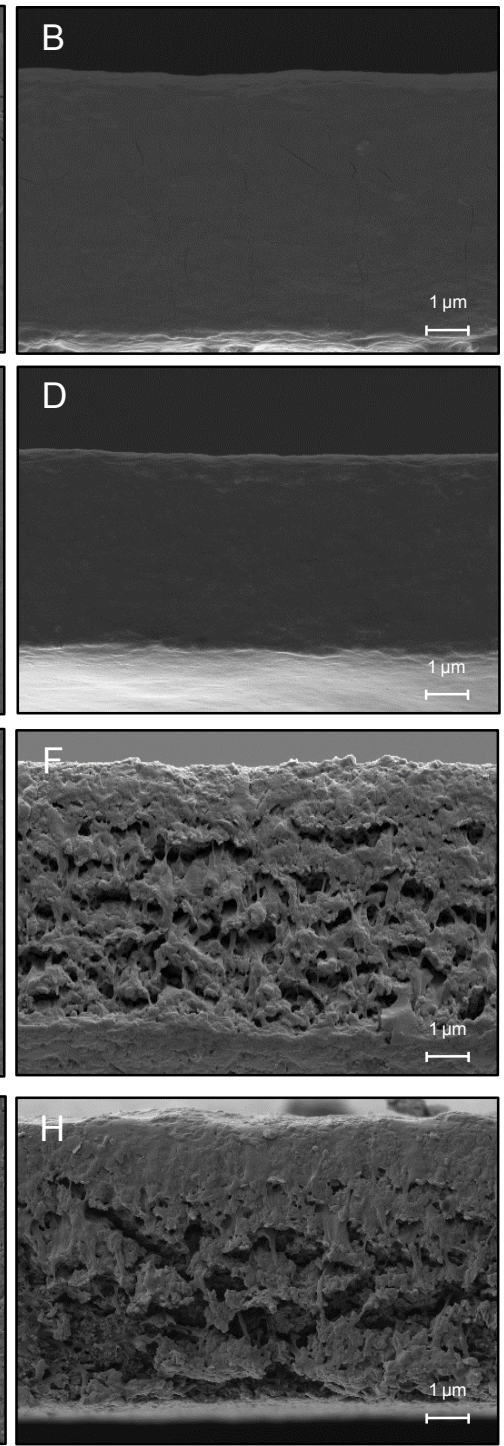

Fig. 2: Surface and cross-section SEM images of each film

Surface SEM images with a magnification of $10000 \mathrm{X}$ and cross-section images with a magnification of $5000 \mathrm{X}$ of each film $\mathrm{A}, \mathrm{B}$. blank FFG1, C,D. blank-FFG2, E,F. AM-FFG1 and G,H. AM-FFG2 

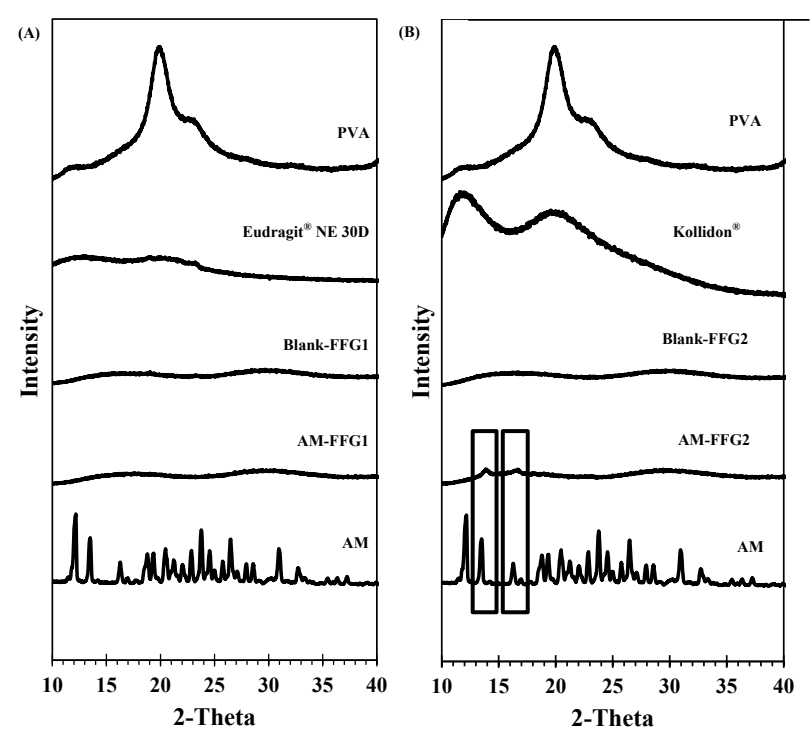

Fig. 3: XRD diffractograms

\section{(A) FFG1 and AM-FFG1, and (B) FFG2 and AM-FFG2}

containing Kollidon ${ }^{\circledR}$ 90F. As a consequence, AM was incapable of rearrange its molecules into an ordered structure, and thus, resulting in an amorphous state. On the contrary, AM-FFG2 containing water soluble Kollidon $^{\circledR}$ 90F could have more time for AM to re-crystalline during the solvent evaporation process. Therefore, the partial crystalline of AM was observed in AM-FFG2 film. In addition, the amorphous nature of AM in the film could be a result from the molecular interaction between AM and film-forming polymer via hydrogen bond which associated with reduced molecular mobility and consequently reduced drug recrystallization $^{[15]}$.

In vitro permeation studies of AM-FFGs were tested using a Franz diffusion cell. AM has poor water solubility of $2.18 \pm 1.32 \mu \mathrm{g} / \mathrm{ml}$. Therefore, the deionized water containing $2 \%$ Tween 80 was used as a receiver medium to ensure the sink condition by increasing its solubility to $89.30 \pm 2.21 \mu \mathrm{g} / \mathrm{ml}$. As shown in fig. 4 , both formulations showed a biphasic permeation profile with a fast permeated characteristic within 30 min followed by a slow permeation up to $8 \mathrm{~h}$. However, only small amount of AM was detected in receiver medium. At the first $30 \mathrm{~min}$, the AM permeated from AM-FFG1 and AM-FFG2 was $9(22.5 \mu \mathrm{g} / \mathrm{ml})$ and $5 \%(12.5 \mu \mathrm{g} / \mathrm{ml})$, respectively. Then, after $8 \mathrm{~h}$, they increased to 13.6 $(27.4 \mu \mathrm{g} / \mathrm{ml})$ and $6.9 \%(17.5 \mu \mathrm{g} / \mathrm{ml})$, respectively. Interestingly, the permeation profiles in epidermis are similarly to the receiver chamber. AM-FFG1 and AMFFG2 showed a rapid AM accumulation within first $30 \mathrm{~min}, 36(90 \mu \mathrm{g} / \mathrm{ml})$ and $26 \%(65 \mu \mathrm{g} / \mathrm{ml})$, respectively. After $8 \mathrm{~h}$, they slowly increased up to $38(95 \mu \mathrm{g} / \mathrm{ml})$ and
$28 \%(70 \mu \mathrm{g} / \mathrm{ml})$, respectively. The biphasic permeation characteristics were due to the conversion of gels into films during application. AM-FFG was applied on the skin as a gels form, which could convert into film with in 30 min, thus, resulting in a fast permeation in the first $30 \mathrm{~min}$. After the gel converted into a film, a slower AM permeation profile was observed. Nevertheless, the prolonged AM permeation profile could be results from the occlusive effect. This phenomenon can reduce the trans epidermal water loss (TEWL) of the skin, leading to induce the swelling of keratinocyte cells and increase in AM permeation ${ }^{[16]}$. In addition, the high amount of accumulated AM in epidermis could be due to the fact that $\mathrm{AM}$ is a highly hydrophobic with a $\log p$ of $4.64^{[17]}$. Therefore, it possesses strong interaction with the membrane ${ }^{[18]}$, resulting in AM accumulation within the epidermis lipid layer. From therapeutic standpoints, a fast permeation of AM is considered advantage as sufficient amount of AM could exert antimicrobial activities followed by a prolonged permeation of AM

As expected, the AM-FFG1 showed a higher cumulative AM permeated in receiver chamber and epidermis in compared to the AM-FFG2. This result is related to their XRD result. AM incorporated in FFG1 showed an amorphous nature, while AM incorporated in FFG2 illustrated a partially crystalline nature. As a consequence, amorphous AM contained in FFG1 could be dissolved faster, and thus, rapidly permeated into the epidermis and receiver chamber than AM contained in $\mathrm{FFG}^{[15]}$.

The antibacterial activity of AM-FFGs against $P$. acnes and $S$. aureus was evaluated. The mechanism of AM against both tested bacteria is well reported. AM could interact with the hydrophobic region of the bacteria

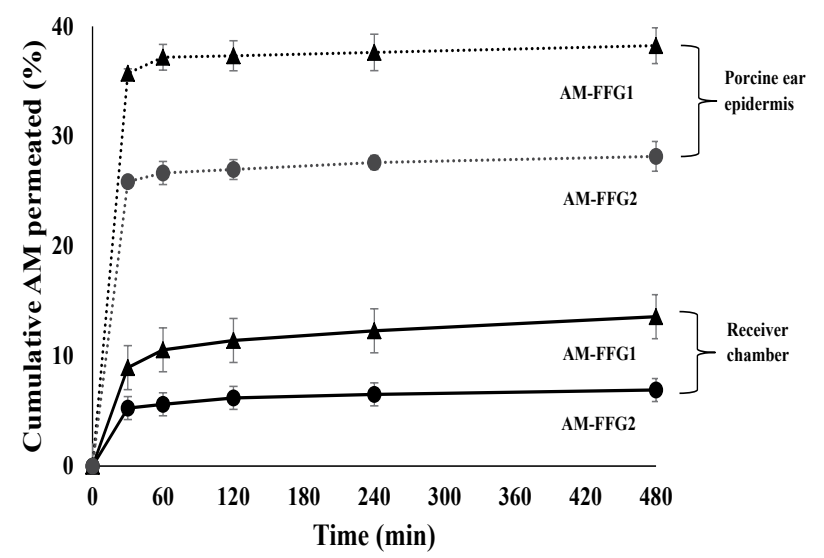

Fig. 4: In vitro permeation profile of AM-FFG1 and AM-FFG2 In vitro permeation profile of AM-FFG1 and AM-FFG2 in receiver chamber and porcine ear epidermis. Data are presented as mean $\pm \mathbf{S D}, \mathbf{n}=\mathbf{3}$. 
membrane and rapidly disrupts the cytoplasmic membrane, thereby, causing leakage of intracellular content ${ }^{[3]}$. The minimum inhibitory concentration (MIC) of AM against $P$. acnes and $S$. aureus were found to be 6.25 and $50 \mu \mathrm{g} / \mathrm{ml}$, respectively ${ }^{[19]}$. Therefore, to ensure antibacterial activity, the AM-FFGs was prepared with $0.125 \% \mathrm{w} / \mathrm{w}$ of $\mathrm{AM}$, which is 5 times greater than its MIC value. Then, the antibacterial activity of AM-FFGs against $P$. acnes and $S$. aureus was evaluated. Both AMFFG1 and AM-FFG2 illustrated antimicrobial activities as evidenced from clear inhibition zone, figs. 5A and B. Nevertheless, the blank FFGs showed no effect against the tested bacteria. Therefore, the antimicrobial activity of AM-FFGs against $P$. acnes and $S$. aureus was resulted from AM. The results showed that AM-FFG2 shows a wider clear inhibition zone, than AM-FFG1. For $P$. acnes AM-FFG1 and AM-FFG2 illustrated a clear inhibition zone of $15.13 \pm 0.15$ and 19.03 $\pm 0.11 \mathrm{~mm}$ (fig. 5A), respectively, and $17.08 \pm 0.20$ and $20.05 \pm 0.13 \mathrm{~mm}$ for $S$. aureus, respectively, fig. 5B. The size of inhibition zone was dependent on a formulation viscosity. The higher viscosity, the slower drug diffuse through the agar ${ }^{[20]}$. AM-FFG2 had a lower viscosity than AM-FFG1, indicating higher drug diffusion ability than another one resulting in a larger inhibition zone.

In addition, the viscosity of AM-FFGs and AM chemical stability were affected by the storage temperature. After 6 mo storage in darkness at room temperature, both AM-FFG formulations showed a slightly increase in the viscosity, fig. $6 \mathrm{~A}$. In contrary, those stored at $4^{\circ}$ showed similar viscosity comparing to initial preparation, fig. 6B. This could be simply explained by kinetic energy of a system increases as the temperature increases, which could lead to the faster ethanol evaporation rate. On the other hand, the chemical stability was also investigated in term of AM remaining. As shown in figs. 7A and $\mathrm{B}$, after 3 mo storage at room temperature, AM-FFG1 and AM-FFG2 showed lower AM remaining (81 and $83 \%$, respectively) than those stored at $4^{\circ}$ ( 83 and $88 \%$, respectively). Nevertheless, after 6 mo storage, AM remaining was slightly decreased under both storage conditions. At room temperature, the AM remaining of AM-FFG1 and AM-FFG2 was 73 and $76 \%$, respectively, while those stored at $4^{\circ}$ showed remaining AM content of 78 and $81 \%$, respectively. AM degradation could be presumed by oxidation reaction as reported by Nishiyama et al..$^{[21]}$. Hence, the addition of antioxidant into AM-FFGs and storage at $4^{\circ}$ would help to improve the chemical stability of AM.

In the present study, AM as a hydrophobic compound was successfully loaded into film-forming gels. The differences in physicochemical properties of each FFGs were affected by various film-forming polymers (Eudragit $^{\circledR}$ NE 30D and Kollidon ${ }^{\circledR} 90$ F). The obtained AM-FFGs showed viscous gel and high AM incorporation efficiency up to $96 \%$. Despite the AM-FFGs contain low amount of ethanol which could help reduce skin irritation, these still would rapidly dry within 30 min after application. AM-FFGs have been formulated to deliver AM to its target site at epidermis with a rapid onset in $30 \mathrm{~min}$, which remained over $8 \mathrm{~h}$. More importantly, these formulations provided excellent antibacterial activity against acne-causing bacteria. AM-FFGs showed good physicochemical stability during storage at $4^{\circ}$ for 6 mo. In summary, the AM-FFGs prepared exhibited high potential to be developed as topical antiacne products.
(A)

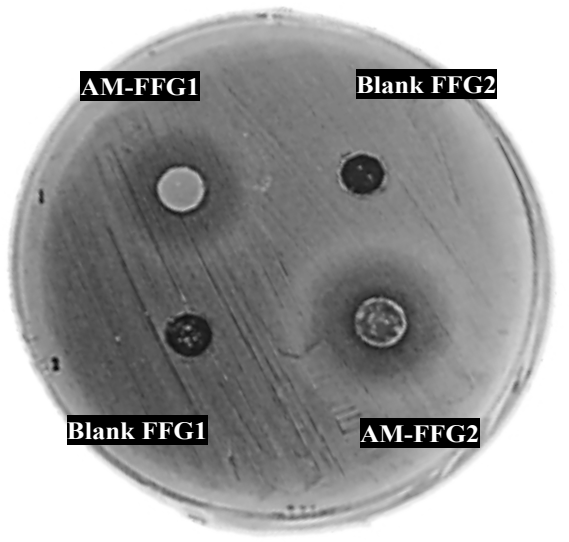

(B)

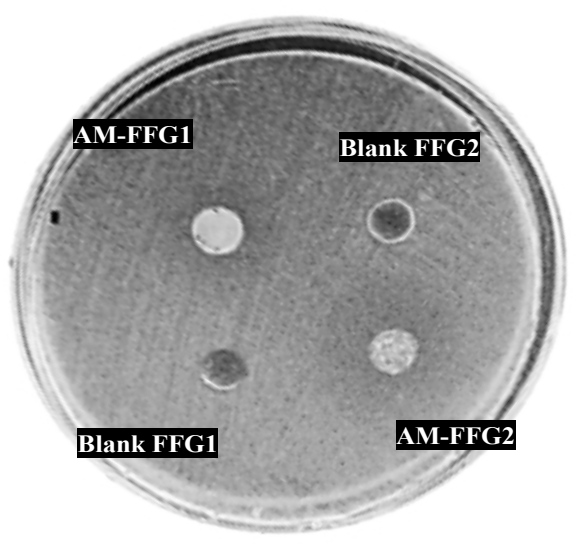

Fig. 5: Antibacterial Activity of AM-FFG1 and AM-FFG2 Antibacterial activity of AM-FFG1 and AM-FFG2 against A. P. acnes and B. S. aureus 
(A)

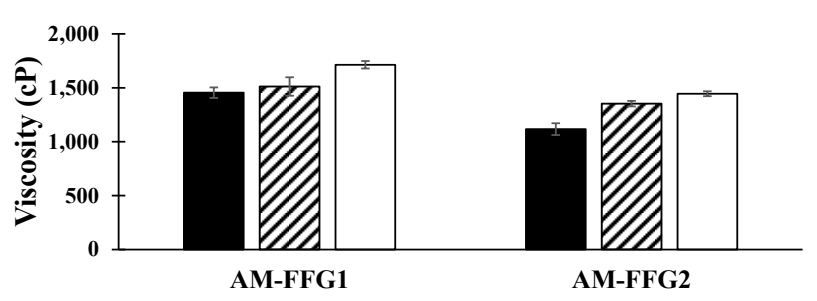

(B)

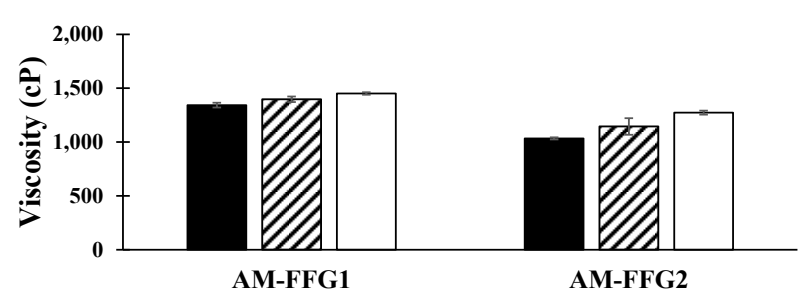

Fig. 6: Viscosity of AM-FFG1 and AM-FFG2 after 3 and 6 months storage

Viscosity of AM-FFG1 and AM-FFG2 after 3 ( $\square$ ) and 6 (//) months storage at $A$. room temperature and $B$. $4^{\circ}$. Data are presented as mean $\pm S D, n=3$, ( $($ ) initial values

(A)

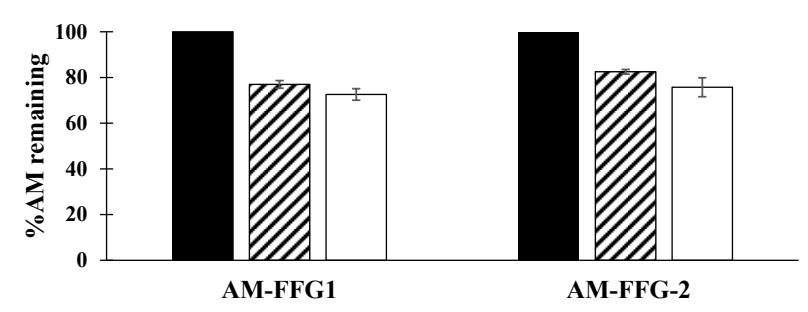

(B)

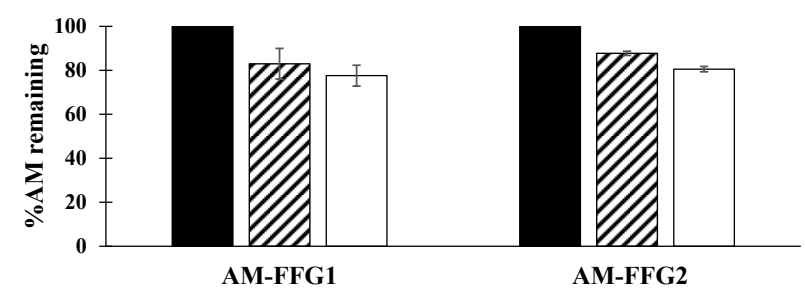

Fig. 7: Percent AM remaining in AM-FFG1 and AM-FFG2 after 3 and 6 months storage

Percent AM remaining in AM-FFG1 and AM-FFG2 stored for 3 ( $\square$ ) and $6(/ /)$ months at A. room temperature and B. $4^{\circ}$. Data expressed as mean $\pm S D, n=3$, ( $\square)$ initial level.

\section{Acknowledgements:}

This research was financially supported by Naresuan University and the National Research Council of Thailand (NRCT) under graduate student program in the fiscal year 2019 (Grant No. 2562/17). Special thanks to the Faculty of Pharmaceutical Sciences and the Faculty of Medical Sciences, Naresuan University for providing the necessary facilities. Also, many thanks to Mr. Paul Freund of the Naresuan University
Language Centre for his editing assistance and advice on English expression in this document.

\section{Conflict of interest:}

The authors declare no conflict of interest.

\section{REFERENCES}

1. Titus S, Hodge J. Diagnosis and Treatment of Acne. Postgrad Med 2012;86(8):734-40.

2. Fox L, Csongradi C, Aucamp M, Du Plessis J, Gerber M. Treatment Modalities for Acne. Molecules 2016;21(8):1-20.

3. Koh J, Qiu S, Zou H, Lakshminarayanan R, Li J, Zhou X, et al. Rapid Bactericidal Action of Alpha-Mangostin against MRSA as an Outcome of Membrane Targeting. Biochim Biophys Acta 2013;1828(2):834-44.

4. Sukatta U, Rugthaworn P. Development of Mangosteen AntiAcne Gel. Kasetsart J Nat Sci 2008;42(5):163-8.

5. Ibrahim MY, Hashim NM, Mariod AA, Mohan S, Abdulla MA, Abdelwahab SI, et al. $\alpha$-Mangostin from Garcinia mangostana Linn: An Updated Review of Its Pharmacological Properties. Arab J Chem 2016;9(3),317-29.

6. Ngawhirunpat T, Opanasopi P, Sukma M, Sittisombut C, Kat A, Adachi I. Antioxidant, Free Radical-Scavenging Activity and Cytotoxicity of Different Solvent Extracts and Their Phenolic Constituents from the Fruit Hull of Mangosteen (Garcinia Mangostana). Pharm Biol 2010;48(1):55-62.

7. Singh V, Kukreti G, Singh MF. Film Formers Gels (FIFOGE ): A Novel Approach. J Pharm Res 2013;1(1):22-8.

8. Bajaj H, Kumar T, Singh VJ. Film Forming Gels : A Review. Res J Pharm Biol Chem Sci 2016;7(4):2085-91.

9. Asasutjarit R, Larpmahawong P, Fuongfuchat A, Sareedenchai V, Veeranondha S. Physicochemical Properties and AntiPropionibacterium Acnes Activity of Film-Forming Solutions Containing Alpha-Mangostin-Rich Extract. AAPS PharmSciTech 2014;15(2):306-16.

10. Zhang ZH, Han Z, Zeng XA, Xiong XY, Liu YJ. Enhancing Mechanical Properties of Chitosan Films via Modification with Vanillin. Int J Biol Macromol 2015;81:638-43.

11. Pan-on S, RujivipatS, Ounaroon A, Kongkaew C. Development, Characterization And Skin Irritation Of Mangosteen Peel Extract Solid Dispersion Containing Clay Facial Mask. Int J Appl Pharm 2018;10(5):202-8.

12. Pothitirat W, Chomnawang MT, Gritsanapan W. Anti-AcneInducing Bacterial Activity of Mangosteen Fruit Rind Extracts. Med Princ Pract 2010;19(4):281-6.

13. Balouiri M, Sadiki M, Ibnsouda SK. Methods For In Vitro Evaluating Antimicrobial Activity: A Review Methods For In Vitro Evaluating Antimicrobial Activity : A Review. J Pharm Anal 2015;6(2):71-9.

14. Schmid-Wendtner MH, Korting HC. The $\mathrm{pH}$ of the Skin Surface and Its Impact on the Barrier Function. Skin Pharmacol Physiol 2006;19:296-302.

15. Sriyanti I, Edikresnha D, Rahma A, Miftahul MM, Rachmawati $\mathrm{H}$, Khairurrijal K. Mangosteen Pericarp Extract Embedded in 
Electrospun PVP Nanofiber Mats: Physicochemical Properties and Release Mechanism of $\alpha$-Mangostin. Int J Nanomedicine 2018;13:4927-41.

16. Well I, Princen MH. Diffusion theory analysis of transepidermal water loss through occusive films. J Soc Cosmetic Chem 1977;28:481-4.

17. Chin GS, Todo H, Kadhum WR, Hamid MA, Sugibayashi K. In Vitro Permeation and Skin Retention of $\alpha$-Mangostin Proniosome. Chem Pharm Bull 2016;64(12):1666-73.

18. Chanburee S, Tiyaboonchai W. Enhanced Intestinal Absorption of Curcumin in Caco-2 Cell Monolayer Using Mucoadhesive
Nanostructured Lipid Carriers. J Biomed Mater Res Part B Appl Biomater 2018;106(2):734-41.

19. Lamlertthon S, Tanngoen P, Tiyaboonchai W. Effects of AlphaMangostin And Citronella Oil Against Acne Causing Bacteria. Int J Pharm Pharm Sci 2019;11(6):45-9.

20. Liu B, Goree J, Vaulina OS. Test of the Stokes-Einstein Relation in a Two-Dimensional Yukawa Liquid. Phys Rev Lett 2006;96:015005-1-4.

21. Nishiyama Y, Amano Y, Ogamino T, Nishiyama S. Oxidation of Mangostins, the Naturally Occurring Xanthone Derivatives Carrying Diverse Biological Activities. Electrochemistry 2006;74:609-11. 